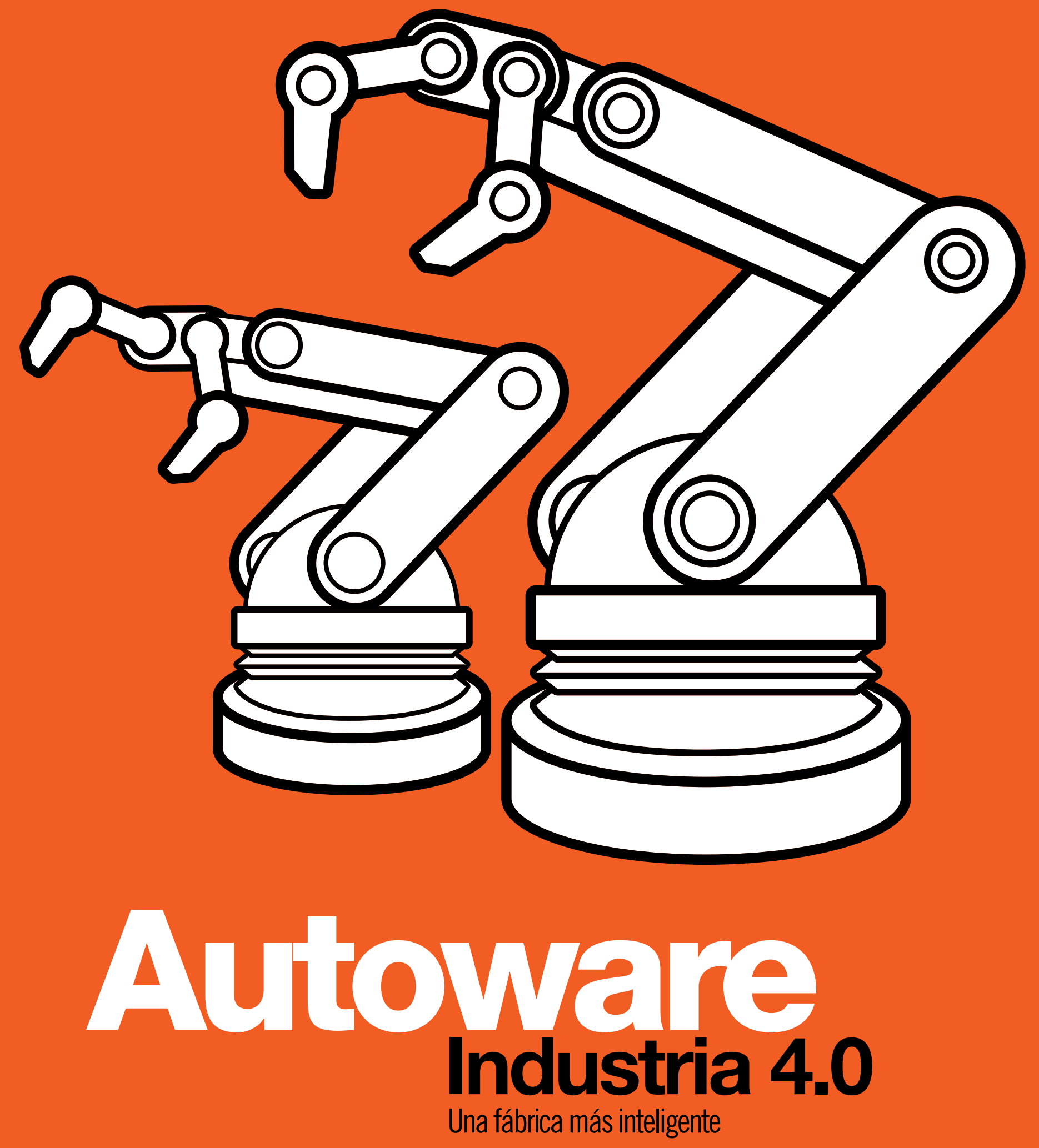




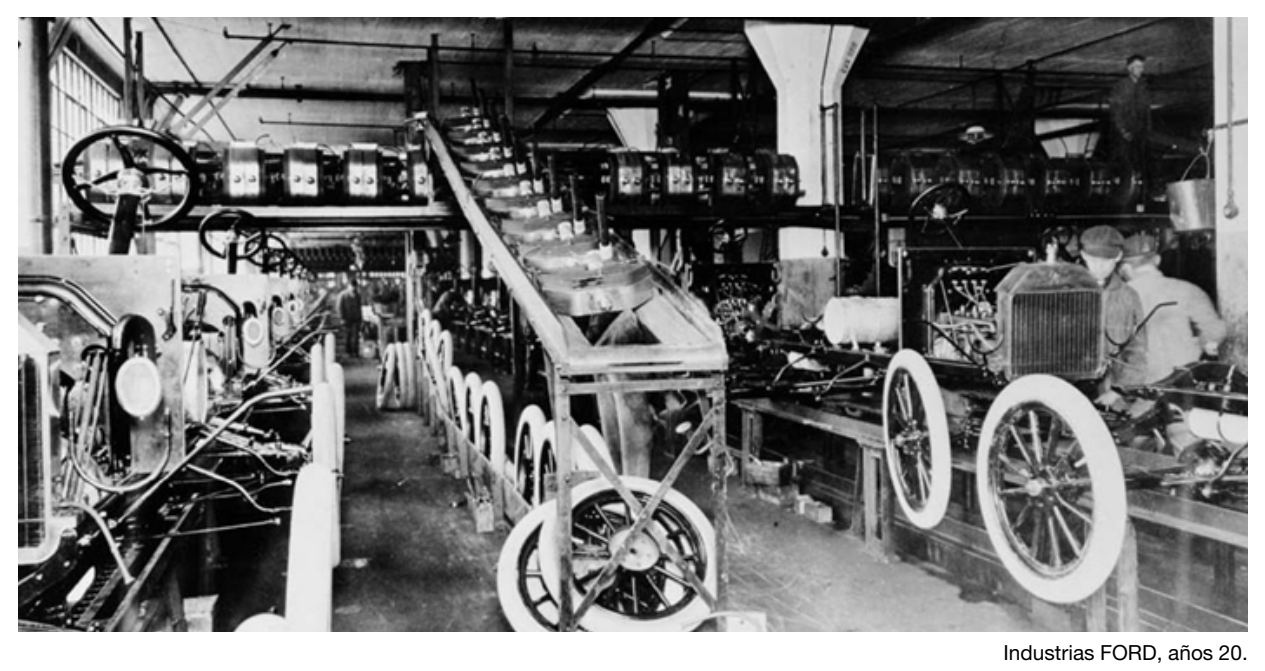

dustria del futuro, de tal manera que todas las compañías adoptarán estas tecnologías para conseguir ser viables. En un escenario de competencia global, las empresas deben ser capaces de responder de forma rápida y económica a los requerimientos del mercado y, para ello, la digitalización de las fábricas será fundamental. De ahí la importancia de las investigaciones en este campo, como es

ALICIA DE LARA

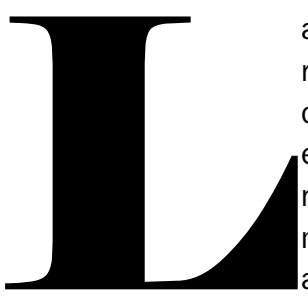

a industria 4.0 hace referencia a un modelo de fábrica en el que la maquinaria no solo está automatizada, sino que, además, trabaja de forma conectada con el fin de mejorar la flexibilidad y capacidad de reconfiguración de los procesos de producción. Para ello, las diferentes máquinas y robots que participan en estos procesos deben ser capaces de intercambiar información relativa a cada proceso (su estado, información recogida de sensores o posibles alertas). Se trata de una fábrica a la que se dota de una mayor inteligencia para alcanzar los siguientes objetivos: ahorrar costes, mejorar la flexibilidad y productividad de los sistemas de producción y reducir los riesgos. Todo ello, a partir de la digitalización de los procesos de producción, del intercambio de información en tiempo real y del desarrollo de sistemas cognitivos capaces de aprender y adaptar la producción.

Esta nueva forma de fabricar se implantará gradualmente y se convertirá en la in- el caso del proyecto AUTOWARE, financiado por la Comisión Europea dentro de la convocatoria H2O2O “Automatización Digital” y en el que participa la Universidad Miguel Hernández (UMH) de Elche.

La colaboración de la UMH en este proyecto corre a cargo del laboratorio UWICORE, especializado en sistemas de comunicaciones móviles e inalámbricas e integrado en el Departamento de Ingeniería de Comunicaciones de la UMH. Dentro del proyecto, el equipo de investigación de la UMH lidera el desarrollo de las redes de comunicaciones inalámbricas industriales, que facilitarán la interconexión entre máquinas (incluidos robots móviles), productos y servicios en la nube, así como el intercambio de datos en tiempo real.

La implementación de redes inalámbricas en los entornos de trabajo permitirá mejorar los sistemas de toma de decisión, detectar fallos o ineficiencias, así como adaptar los procesos de producción con mayor rapidez y agilidad, tal y como explica el profesor del Área de Teoría de la Señal y Comunicaciones Javier Gozálvez. Según el investigador, en la actualidad, los sistemas de producción tienen una conectividad limitada y la capacidad de adaptación y corrección de errores depende, en gran medida, de la intervención humana: "Por ejemplo, pensemos en una cadena de producción en la que cada máquina tiene su función. Si se produce un fallo en una -porque por ejemplo es descalibrada por un golpe fortuito- entonces aunque la producción no se interrumpa,

el fallo afectará a la calidad de las piezas, que acabarán siendo desechadas mientras el fallo no sea detectado". Javier Gozálvez añade que, en la actualidad, la capacidad de reconfiguración o adaptación de los sistemas de producción no es todo lo flexible que sería deseable. "Para dar solución a estos inconvenientes se trabaja desde el proyecto AUTOWARE", subraya el experto.

\section{Fábricas digitalizadas}

Según datos de la Unión Europea, la digitalización de productos y servicios aportará a la industria más de 110.000 millones de euros al año en Europa durante los próximos 5 años. Solo en Alemania, el país europeo 


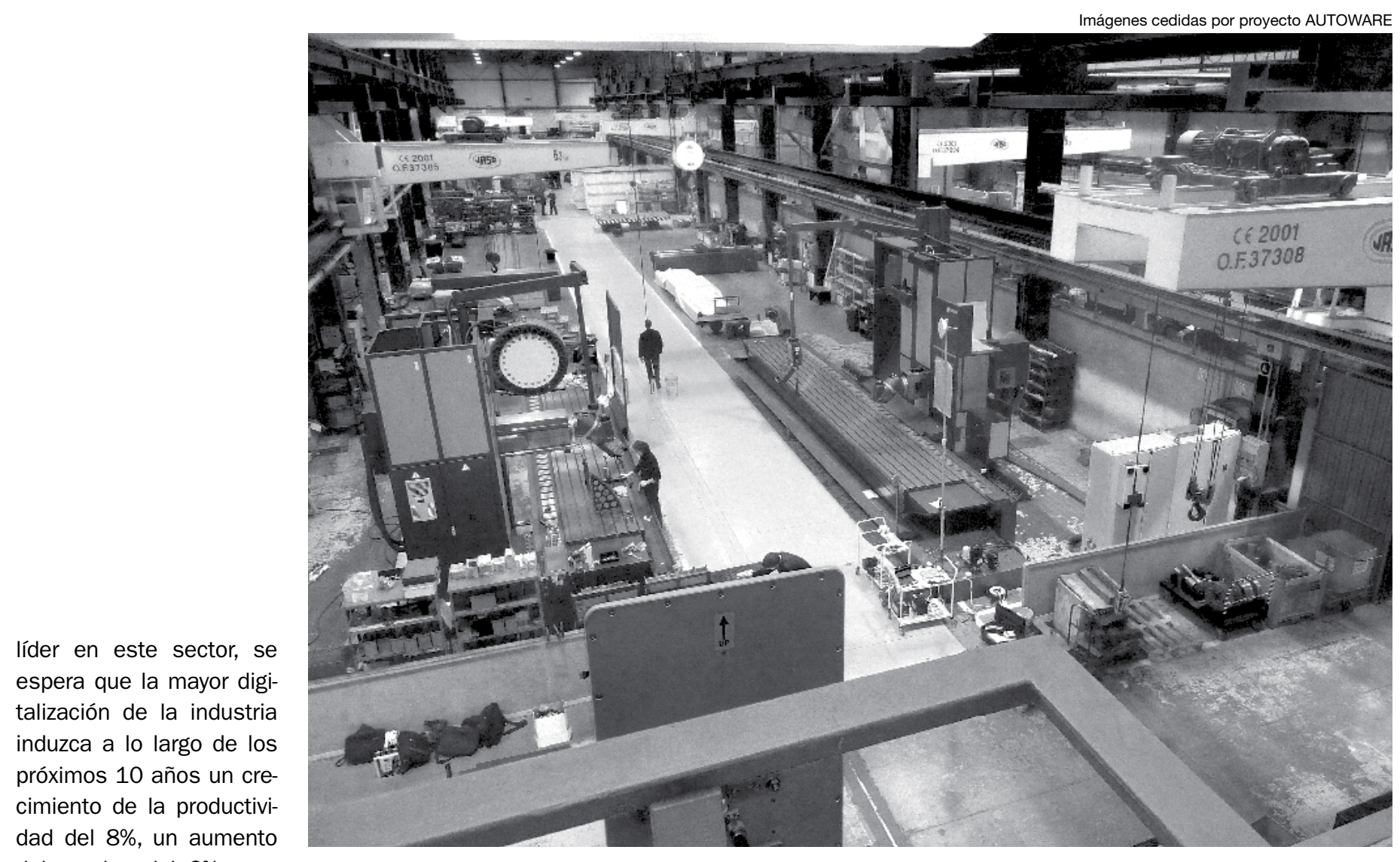

del empleo del $6 \%$ y un

ascenso de los ingresos de unos 30.000

millones de euros al año. Esta revolución,

en la que otros países ya se encuentran in-

mersos, se presenta como una gran opor-

tunidad de transformarse

y ganar una posición com-

petitiva reforzada para paí-

ses como España, según

\section{"En la Industria 4.0, cada uno de los procesos estará digitali- zado", señala Gozálvez}

el propio Ministerio de In-

dustria, Energía y Turismo.

El profesor Gozálvez subraya que, a pesar de que las fábricas actuales están robotizadas, la fábrica no está digitalizada y la conectividad de los robots y

ductos desechados, con el consecuente ahorro", matiza Gozálvez.

La UMH es la única universidad integrada en el consorcio del proyecto y su tarea se centra en máquinas es limitada. Ta y como puntualiza el docente, con la tecnología que implementará la industria 4.0, cada uno de los procesos estará digitalizado y se detectará en tiempo real si ha habido algún error y se podrá corregir de una forma dinámica y flexible. "Será posible obtener un informe específico de cada uno de los procesos para verificar que cada parte se ha ejecutado correctamente y proporcionar el reajuste necesario para corregir el funcionamiento de la cadena en caso de fallo", explica el investigador. $Y$ todo esto conectado con la nube o con otros nodos locales o remotos para procesar toda la información digital que las fábricas del futuro generarán. "Una rápida detección de errores a través de la digitalización y conexión de la fábrica permitirá reducir la cantidad de pro- la mejora de la conectividad entre los dispositivos a través de redes inalámbricas. Un avance importante, puesto que, hasta el momento, la conectividad en las fábricas se lleva a cabo principalmente a través de cable. Sin embargo, como explica la también investigadora de la UMH $\mathrm{M}^{\mathrm{a}}$ Carmen Lucas, contratada en el proyecto AUTOWARE, el cable limita la capacidad de reconfiguración de la fábrica y no permite la conectividad entre robots móviles porque dificultaría su desplazamiento por las plantas, algo que sí posibilita las redes inalámbricas.

Asimismo, Lucas señala que la implementación de la red inalámbrica en la industria conlleva cumplir con unos estrictos requisitos de fiabilidad, retardo y de velocidad de transmisión, impuestos por las aplicaciones industriales. Es decir, "la información sobre un posible fallo en la cadena de producción debe transmitirse en un tiempo mínimo y debe hacerse de una forma fiable para alcanzar los requerimientos exigidos", subraya la investigadora. Además, el grupo de la UMH también se centra en optimizar las capacidades de las redes, algo que consiguen estableciendo predicciones, basadas en análisis de datos, sobre los diferentes procesos que tienen lugar en la fabricación. De esta manera, según explica $\mathrm{M}^{\mathrm{a}}$ Carmen Lucas, se consigue optimizar los recursos de comunicación y garantizar los requisitos de fiabilidad, retardo y velocidad de transmisión: “Las redes inalámbricas industriales desempeñan un papel clave en la reducción de costes y tiempo", subraya.

La conectividad de las fábricas conlleva los riesgos propios de la seguridad, protección y acceso a los datos, tal y como apunta el profesor de la UMH Miguel Sepulcre, quien también colabora en el proyecto. En relación a la seguridad, Sepulcre explica que hay todo un campo de desarrolladores y expertos trabajando en reducir la vulnerabilidad de los 


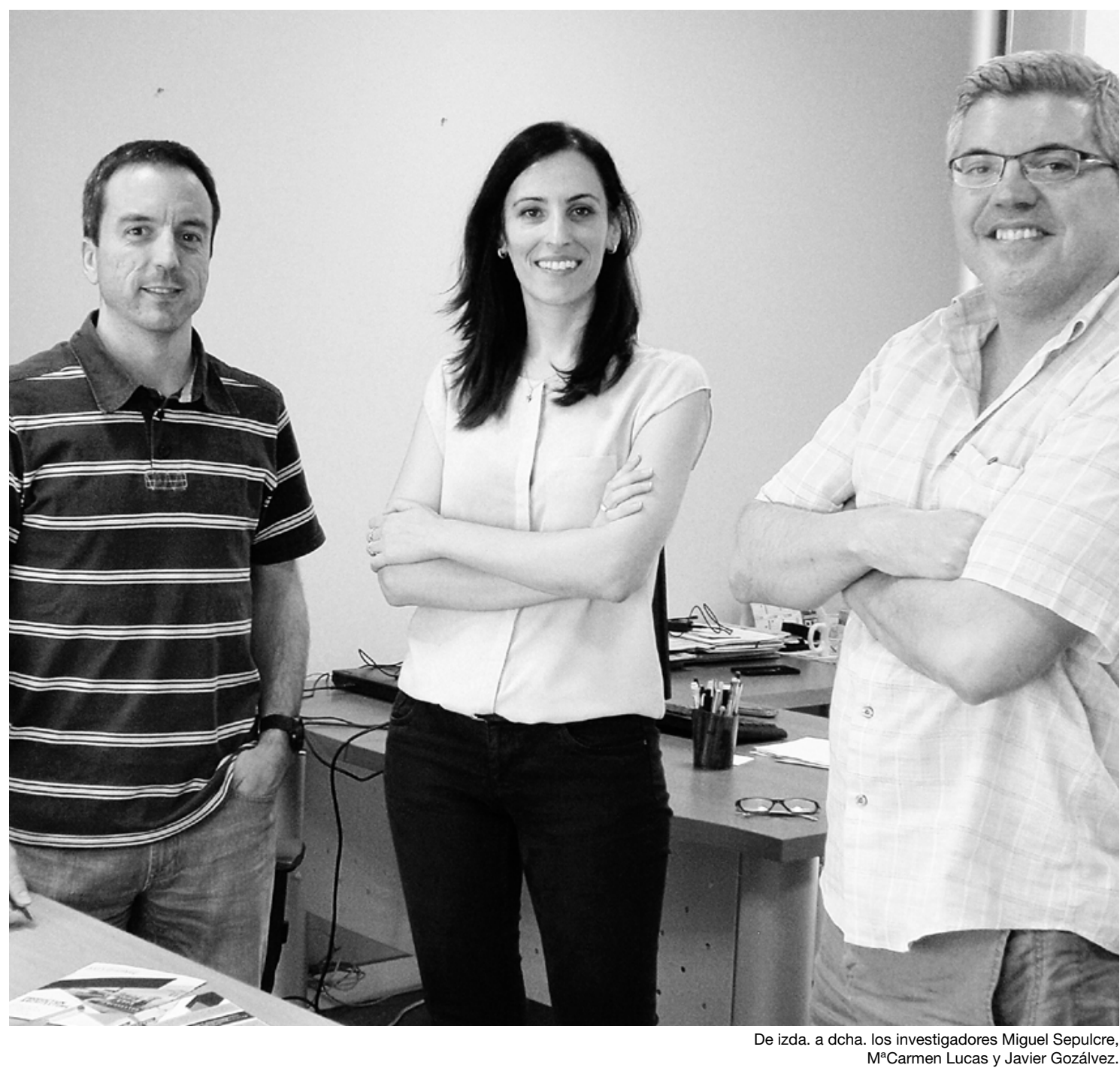

Los profesionales de las telecomunicaciones afrontan los retos que plantea la nueva industria 4.0 en la que su trabajo es y será decisivo. Como explica el profesor del Área de Teoría de la Señal y Comunicaciones Javier Gozálvez, es importante transmitir a la sociedad la versatilidad de los ingenieros de Telecomunicación, cuyos conocimientos y habilidades tienen un impacto notable no sólo en los propios sistemas de telecomunicación (como radio, televisión o teléfono), sino también en la automoción, los sistemas médicos, la banca o la seguridad, por ejemplo.

sistemas, con el objetivo de diseñar redes seguras. Además, como añade $\mathrm{M}^{\mathrm{a}}$ Carmen Lucas, la digitalización de las fábricas puede minimizar muchos otros riesgos, incluidos los laborales. Y de esta manera, "Ias ventajas que ofrece la conectividad a la industria son mayores que los riesgos", coinciden en apuntar los tres expertos.
Las soluciones tecnológicas que se desarrollen en AUTOWARE serán evaluadas en diferentes empresas del consorcio, como la alemana SmartFactory, una planta piloto dedicada a desarrollar y probar los pilares tec- nológicos fundamentales de la Industria 4.0. Según señala el profesor Gozálvez, el proyecto se encuentra en el primero de sus tres años de ejecución y las pruebas piloto de redes inalámbricas en las que trabaja el equipo ilicitano se realizarán más adelante, en el centro tecnológico con sede en Guipuzkoa IK4-TEKNIKER. El objetivo de estos tests se centrará en demostrar los beneficios de la conectividad inalámbrica de robots móviles y fábricas, y la capacidad de garantizar la fiabilidad y baja latencia de las comunicaciones.

El proyecto, que cuenta con una financiación en torno a los 4 millones de euros y se coordina desde España, también está integrado por las siguientes empresas y centros de investigación: SQS e Innovalia (España); SmartFactory, Fraunhofer y SMC (Alemania); TTTech (Austria); Blue Ocean Robotics (Dinamarca); Robovision e Imec (Bélgica); PWR Pack (Holanda); CNR (Italia) y Institut Jozef Stefan (Eslovenia). Un total de 14 socios, que investigan de forma simultánea en las diferentes áreas implicadas en la fabricación cognitiva, para alcanzar modernos sistemas de control de producción que permitan a las fábricas reaccionar de forma autónoma y flexible. Una industria conectada, en la que los robots no solo desempeñarán el papel de simples herramientas de fabricación, sino que también serán capaces de reconfigurarse, corregir fallos y adaptarse a las necesidades de la fabricación. 